\title{
Theoretical evaluation of partial credit scoring of the multiple-choice test item
}

\author{
Rasmus A. X. Persson ${ }^{\mathrm{a}}$ \\ a Department of Pedagogical, Curricular and Professional Studies, University of Gothenburg, \\ Sweden
}

\section{ARTICLE HISTORY}

Compiled November 21, 2021

\begin{abstract}
In multiple-choice tests, guessing is a source of test error which can be suppressed if its expected score is made negative by either penalizing wrong answers or rewarding expressions of partial knowledge. Starting from the most general formulation of the necessary and sufficient scoring conditions for guessing to lead to an expected loss beyond the test-taker's knowledge, we formulate a class of optimal scoring functions of which that due to Zapechelnyuk (Economics Letters, 132, 2015) appears as a special case. We then consider an arbitrary multiple-choice test taken by a rational test-taker that knows an arbitrary fraction of its keys and distractors as a model of a test of factual knowledge. For this model, we study the statistical properties of the obtained score for both standard marking (where guessing is not penalized), and marking where guessing is suppressed either by expensive score penalties for incorrect answers or by different marking schemes that reward partial knowledge.
\end{abstract}

\section{KEYWORDS}

Error variance; multiple-choice test; partial credits model; guessing

\section{Introduction}

Multiple-choice tests are common in educational achievement testing at all levels [see e. g. Lesage, Valcke, and Sabbe (2013) and references therein]. The extra complexity, relative to open questions, in item construction is for large numbers of test subjects offset by the ease of scoring. The objective and transparent scoring also provides the students with a sense of fairness in the examination. Moreover, the tests are quick to administer and can thus cover a wide breadth of the topic examined. Nevertheless, the variance in the number of correct answers due to random guessing is appreciable (Burton \& Miller, 1999; Zimmerman \& Williams, 1965, 2003), and this confounds the task of ranking test-takers according to their "true score" (a theoretical measure of their ability free from measurement errors).

Assuming unbiased guessing among all the answer alternatives, for each item with $c$ options that the test-taker guesses, the ideal test-retest variance increases additively by $\frac{1}{c}\left(1-\frac{1}{c}\right) \cdot{ }^{1}$ Prima faciae, the variance would thus be expected to be greatest for

CONTACT R. A. X. Persson. Email: rasmus.persson@gu.se

${ }^{1}$ Assuming that the test-taker has gained or lost no knowledge since the last test, and does not remember what answers she has provided earlier. 
the student of lowest ability for whom guessing is a prominent part of the test-taking strategy (and this is also proven to be the case in a more careful analysis). However, partial knowledge may lead to the exclusion with certainty of one or more given alternatives. This has in theory the effect of decreasing the apparent value of $c$, and hence increasing the test-retest variance (in addition to the expectation value of the score). The influence of partial knowledge on the score is apparent, for instance, in the study by Medawela, Ratnayake, Abeyasinghe, Jayasinghe, and Marambe (2018) on dentistry students where parallel forms of multiple-choice and fill-in-the-blanks tests were used.

Whereas computerized forms of multiple-choice testing where the answer options are presented one-at-a-time may mitigate the problem of error variance due to guessing (Papenberg, Diedenhofen, \& Musch, 2021), guessing remains a rational strategy (and is in any case impossible to implement in pencil-and-paper forms); this applies also to the variation where the test-taker is allowed "answers until correct" (Wilcox, 1981). Another way to address the problem is to subtract points for incorrect answers, a procedure sometimes called "formula scoring" (Budescu \& Bar-Hillel, 1993; Lord, 1975). This provides strong incentive not to guess when in doubt but may however entail a perceived problem of "fairness" in the test-taker population. Additional variance due to differences in risk-taking behavior is also introduced (Diamond \& Evans, 1973), and an "intimidation factor", detrimental to the test validity, associated with this type of scoring has been reported (Betts, Elder, Hartley, \& Trueman, 2009; Bliss, 1980). Although this latter effect can be psychologically mitigated, and maybe removed entirely, by providing points for skipped questions instead of subtracting points for incorrect answers (Prieto \& Delgado, 1999; Traub, Hambleton, \& Singh, 1969).

It has also been suggested (Akeroyd, 1982; Arnold \& Arnold, 1970; Bradbard, Parker, \& Stone, 2004; Bush, 2001; Coombs, Milholland, \& Womer, 1956; Dressel \& Schmid, 1953; Frandsen \& Schwartzbach, 2006; Gibbons, Olkin, \& Sobel, 1979; Otoyo \& Bush, 2018; Slepkov \& Godfrey, 2019; Zapechelnyuk, 2015, among others) that partial marks should be awarded to test-takers who indicate correctly that they know some options are wrong, rather than hazard a guess on the right answer. The added complexity of choice does not seem to pose any significant problems for the test-takers (Ben-Simon, Budescu, \& Nevo, 1997). Besides the greater discriminatory power that the test is supposed to achieve like this by extending the effective scoring range, it is also an instrument to penalize blind guessing by rewarding the expression of partial knowledge.

The fundamental problem with scoring systems of partial knowledge is that there is arbitrariness in how to award partial credit. While some authors derive scoring systems from different axioms (Frandsen \& Schwartzbach, 2006; Zapechelnyuk, 2015), such an approach ignores the question of the actual validity of the test and may like this lead to unintended results, no matter how simple or appealing the underlying axioms are. In general, moreover, corroborating tests and their scoring systems against real-world performance is a time-consuming and expensive endeavor. For instance, the results of Vanderoost, Janssen, Eggermont, Callens, and De Laet (2018) are limited to the particular partial scoring model [that is, the one due to Arnold and Arnold (1970)] evaluated in their study.

In regards to the above-mentioned considerations, in this paper, we present a formalization of the probability of solution of a multiple-choice test as a function of the fraction of the material that the test-taker is presumed to know, and use it to investigate theoretically the characteristics of a number of different scoring systems for the multiple-choice test taken by a rational test-taker striving to maximize her score. Our aim and methods are similar to those of Chica and Tárrago (2021) and of Frary 
(1982), but - save for the occasional numerical integral - here we rely on analytical results only without recourse to numerical simulations.

A limitation of the present study is that we exclude from consideration scoring systems without a discrete set of allowed responses to each item, but where the test-taker is instructed to indicate along a continuum her personal degree of confidence that each alternative is correct (Dirkzwager, 2003; Finetti, 1965; Shuford, Albert, \& Massengill, 1966). These do not fit in general within the model that we will present because the score then represents a $c$-dimensional vector, rather than a one-dimensional latent trait. Let us nevertheless briefly make some observations in their regard before we move on.

Finetti's (1965) proposed quadratic scoring rule, which is a function not only of the confidence expressed in the key but also of those in the distractors, rigorously penalizes guessing but counterintuitively leads to lower scores when distractors are eliminated (for a constant certainty in the key). If, on the other hand, the score is a function only of the confidence expressed in the key, a logarithmic scoring function is the only mathematically admissible one (Dirkzwager, 2003; Shuford et al., 1966). However, this scoring function comes at a cost of unbounded score penalties for erroneous answers which cannot be removed through a transformation to "points for blank answers" to increase test validity as discussed above. Moreover, Dirkzwager (2003) advocates statistical corrections for the self-estimated abilities and a computerized testing form with feedback where the test-taker is gradually trained to estimate her ability more accurately. However, in this way, not only is the transparency in the scoring lost (as well as much of the simplicity of constructing a multiple-choice test), but "correcting" the self-assessed probabilities from observed performance turns guessing into a rational strategy.

\section{Latent-score model}

\subsection{Partial knowledge}

Throughout this paper, we adopt an axiomatic approach in that the latent score should be defined transparently a priori and not extracted statistically a posteriori. This means that we seek a definition of "partial knowledge" that can be evaluated independently of the multiple-choice test and which is intuitively obvious. The axiomatic approach further implies that we can only provide justification for our definition by way of example.

Consider now the following hypothetical test item:

Which is the French word for cat?

A. chien B. chat C. choux D. chouette

A test-taker that knows the meaning of any of the distractors (chien, choux, chouette) can readily rule them out. This we take to be a reflection of the test-taker's "partial knowledge". If she honestly indicates, for instance, that "choux" and "chouette" are not the correct alternative, she has provided evidence that she knows the English meaning of these two words (for else how could she know they are not correct?). If asked, in a parallel form of the test with constructed responses, to give the English meaning of each of these four alternatives, she would succeed at two and fail at the others.

Consider now another example [based on one by Masters (1982)]: 
Which is the capital of Spain?

A. Milano B. Lisbon C. Madrid D. Barcelona

If the test-taker knows that Milano is in Italy, or that Barcelona is in Spain but does not hold the status of national capital, then she could rule these out, thereby making use of her "partial knowledge". To capture this knowledge in a constructed-response parallel form, the test-taker could be asked for each of these cities in turn to provide their country of location and status (capital or not). From her answers, it should be clear whether her knowledge is sufficient to rule them out in this example item. ${ }^{2}$

At this point, we have to consider another type of multiple-choice test item, for which the concept of "partial knowledge" appears ill-defined, for instance, an item like:

Which of the below options is a synonym of "tenebrous"?

A. happy B. sad C. bright D. dark

Quite clearly, the test-taker might know the meaning of each of the four alternatives and still not know the meaning of "tenebrous" used in the item stem. But if the item is reformulated, for instance, by asking "Pick the two words below that are synonyms", partial knowledge can be used to the test-taker's advantage by the method of exclusion. A parallel form with constructed responses for this last variant of the test would ask for synonyms of each of the alternatives in turn.

From these examples, we propose the following definition.

Definition 2.1. For tests of vocabulary or factual knowledge more generally, provided that all of the terms in the stem of the multiple-choice test item are known and understood, the "latent score" on the item can be ascertained by the number of correct responses on a parallel constructed-response form where the test-taker is quizzed in isolation on the meaning or implications of each of the answer options, if such a parallel form can be constructed.

Note that "knowledge" about a distractor extends in our definition also to recognizing it as a made-up word; in fact, anything that makes it possible to rule it out is to be considered "knowledge" (and analogously for the key). ${ }^{3}$ In the most general case, the test-taker is expected to answer, with full credit, all items correctly for which she knows either the meaning (and implications) of the key, or for which she knows the same for all of the distractors; and to answer, with partial credit, all other items for which she has only partial knowledge of the answer options. Keeping a general approach, by "partial credit" we consider also "no credit" as a special case, as long as the answer is not completely erroneous (i.e., the correct option not indicated at all). Nevertheless, it should be clear - and we will return to this point in a subsequent section - that our definition of the latent score (that is, as determined by the parallel constructed-response form of the multiple-choice test) cannot map perfectly to any partial credit system for the multiple-choice test item. For instance, providing the correct answer to an item can be the result of luck, of knowing the key but no distractors, of knowing all of the distractors but not the key, or of knowing all distractors and key.

\footnotetext{
${ }^{2}$ In fact, simply asking the test-taker a series of open questions of the form "tell us about X", where X is an answer option, should suffice.

${ }^{3}$ In a test of mathematics, a test-taker may only be able to arrive at a partial solution of the problem; however, the rational test-taker that is aware of this limitation will then exclude this answer option, if present, when guessing the correct answer, leading to an inversion of the credits model we consider. This is a situation that may arise in test items where the solution requires the progression through several steps and will not be considered in this paper.
} 
Like Dirkzwager (2003), the type of multiple-choice test item that we will consider is one where there is only one correct answer option. An analysis of the case where each item has multiple keys is presented in a companion paper (Persson, 2021). We shall consider an item to be correctly answered if only the one correct option is indicated; if the correct option is indicated in addition to other incorrect ones, the answer is only partially correct. Otherwise, it is incorrect. It follows from these definitions that the blank answer is partially correct (since it is equivalent to indicating all answer options).

\subsection{Formalization}

Since we are only interested in test statistics, the actual content is immaterial and the model can be readily formalized as follows. First, we consider that an answer alternative is "known" if the test-taker is certain that it is either key or distractor, without needing to know the other alternatives. An alternative is "unknown" if the test-taker is uncertain about its status (we do not distinguish between different levels of uncertainty here). Second, we define a hypothetical "test" by assuming it contains a fixed number of items with $c$ alternatives each, all of which are unique and only appear once in the whole test. Third, we consider a set of "test-takers", each of whom knows a fixed but individual number of concepts drawn randomly from the keys and distractors of the hypothetical test. We may thus consider an arbitrary number of test-takers taking any number of arbitrarily designed tests using random distributions to mimic real-world test-taking populations of any desired statistical characteristics.

Let us denote the probability that the test-taker that masters a fraction $f$ of the material (that is, knows a fraction $f$ of all keys and distractors on the test if queried on a parallel form, which is our definition of the latent score) knows the answer to an item with $c$ options with complete certainty as $P_{1}(f, c)$. Likewise, we denote the probability that she knows with absolute certainty the key to be one of two options, but not which one, as $P_{2}(f, c)$, and so on. The total probability of providing the correct answer, for a randomly chosen pair of rational test-taker and item is (Horst, 1933, rewritten here in differential form)

$$
P(f, c)=P_{1}(f, c)+\frac{P_{2}(f, c)}{2}+\ldots+\frac{P_{c}(f, c)}{c}
$$

where each term corresponds in turn to the probability of guessing the correct option among $1,2, \ldots, c$ alternatives, having excluded $c-1, c-2, \ldots, 0$ of the answer options by partial knowledge. In the Appendix, we show that

$$
P_{1}(f, c)=\sum_{i=0}^{c-1}\left(\begin{array}{c}
c-1 \\
i
\end{array}\right) f^{i+1}(1-f)^{c-i-1}+f^{c-1}(1-f)
$$

and

$$
P_{k}(f, c)=f^{c-k}(1-f)^{k}\left(\begin{array}{c}
c \\
k
\end{array}\right) \prod_{i=0}^{c-(k+1)} \frac{1}{c-i}
$$


for $1<k<c$, as well as $P_{c}(f, c)=(1-f)^{c}$. Here

$$
\left(\begin{array}{c}
c \\
n
\end{array}\right)=\frac{c !}{n !(c-n) !}
$$

is a binomial coefficient.

\subsection{Relation to the Rasch latent-trait model}

Although we will find no use for it, for completeness, we here point out the mathematical relation between our formalization and the Rasch model (Rasch, 1960). These two approaches differ in their assumptions; our formalization makes stronger assumptions and, correspondingly, is only applicable to the type of factual recall discussed above (with no shared keys or distractors between items). On the other hand, we can make stronger a priori predictions through eqs (2) and (3), for this particular type of knowledge tests, than the more general but less committal treatment of Rasch theory, or item response theory more generally, which includes more parameters.

In the Rasch model (Rasch, 1960), each test item is associated with an intrinsic difficulty $\delta$, and the probability of success, $P$, on the item is given by,

$$
\ln \frac{P}{1-P}=\theta-\delta
$$

where $\theta$ is the inherent ability of the test-taker, related in our model to the parameter $f$, whereas the difficulty parameter $\delta$ is a constant (since there is no intrinsic variation in difficulty across items in our formalization; in other words, the test items do not form a Guttman scale). For dichotomous scoring, we have the function $\theta(f)$ through this equation upon substituting the expression for $P(f, c)$ in eq. (1) if guessing is part of the test-taker's strategy, or the expression for $P_{1}(f, c)$ in eq. (2) in case it is not. ${ }^{4}$ Since $P(f, c)$ is a monotonous function of $f, \theta(f)$ is invertible, and so eq. (5) represents a coordinate transformation between $f$-space and $\theta$-space for the latent ability. One might be tempted to conclude that if, in a Rasch analysis, $\delta$ is not found constant across the test items, the assumptions of our formalization do not hold for that particular test; but one must keep in mind that because of measurement error alone, $\delta$ will never be found precisely constant for any real-world data. Nevertheless, having error bounds on $\delta$ allows us to say with some confidence whether the formalization is applicable or not.

The preceding discussion might seem to indicate that the model is very restrictive in its domain of application. However, if $\delta$ is not constant, one may simply group the items by difficulty and apply the analysis to each group individually, each group of items being thus considered as an individual test. One should nevertheless not make the mistake to take the Rasch model as somehow "intrinsically" correct: indeed, for an item that tests only knowledge, it is difficult if not impossible to interpret the "difficulty" as an intrinsic property of the item (unlike, say, for items that requires computations or reasoning, which quite clearly require varying levels of "mental energy"). Without a model representation of the difficulty, it becomes simply a fitting parameter.

In fact, since the test items do not form a hierarchy of difficulty (Rasch difficulty parameters constant), the test is not unidimensional in the traditional sense of psy-

${ }^{4}$ The extension to the polytomous Rasch model (Andrich, 1978; Masters, 1982) follows analogously with $P_{k}(f, c)$. 
chometrics. This means that the computed reliability coefficient is invariant to the addition of more test items within the model. Simply put, in the formalization that we apply, the $N$-item test has $N$ orthogonal dimensions in factor analysis. This is a peculiarity of the mathematical model that we employ, and it can be interpreted as the model of a "pure" achievement test (as opposed to "ability tests" which measure some underlying mental ability).

\section{Error-minimizing scoring function}

Let $p_{n}$ denote the points awarded for a providing $n$ answer options, of which one is correct, to a multiple-choice test item. In other words, $p_{n}, n>1$ designates the point value of a partially correct answer and $p_{1}$ denotes the point value of a fully correct one. If there are $c$ alternatives (one key and $c-1$ distractors), the expected value due to randomly guessing $n$ answer options is then

$$
E(c, n)=\frac{\left(\begin{array}{c}
c-1 \\
n-1
\end{array}\right)}{\left(\begin{array}{c}
c \\
n
\end{array}\right)} p_{n}
$$

Likewise, the score variance when guessing $n$ answer options is given by,

$$
V(c, n)=\left(\frac{\left(\begin{array}{c}
c-1 \\
n-1
\end{array}\right)}{\left(\begin{array}{c}
c \\
n
\end{array}\right)}-\frac{\left(\begin{array}{c}
c-1 \\
n-1
\end{array}\right)^{2}}{\left(\begin{array}{c}
c \\
n
\end{array}\right)^{2}}\right) p_{n}^{2}
$$

The task is to choose the set of coefficients $\left\{p_{i}\right\}_{i=1}^{c}$ so as to minimize the variance whenever the test-taker tries to maximize the expectation value.

\subsection{Necessary and sufficient conditions to suppress guessing}

The strategic choices that the test-taker makes will depend on her knowledge, which is unknown to the test-maker at the time of test construction. Assume that faced with a question item, the test-taker uses her knowledge to narrow the feasible options down to $i$ alternatives. We want to ensure that she provides a partial answer comprising not more and not fewer than these $i$ alternatives, since otherwise an element of chance unrelated to her knowledge is introduced in the score (increasing the testretest variance). Therefore, we require that $E(i, i)>E(i, m)$ for $m<i$ (penalizing the gamble of answering more narrowly than warranted by partial knowledge) as well as $E(i, i)>E(i+1, i+1)$ for $i<c$ (penalizing the "hedging-your-bets" strategy of answering more inclusively than warranted).

The first inequality leads to the condition:

$$
E(i, i)>E(i, i-1)>\ldots>E(i, 1)
$$


A natural way to satisfy it is to write the recursive relation:

$$
p_{i}=\frac{(i-1) p_{i-1}}{i}+\epsilon_{i}, \text { for } i=1, \ldots, c
$$

where $\left\{\epsilon_{i}\right\}$ are positive constants. This recursive relation can be rewritten in closed form as,

$$
p_{k}=\sum_{j=1}^{k} \frac{j \epsilon_{j}}{k} .
$$

The second inequality then leads to the requirement,

$$
\frac{k+1}{k} \sum_{j=1}^{k} j \epsilon_{j}>\sum_{j=1}^{k+1} j \epsilon_{j}
$$

where $c>k \geq 1$. This imposes an upper limit on the values of $\left\{\epsilon_{i}\right\}$.

The ideal test-retest variance due to guessing is eliminated for the rational or riskaverse test-taker with all scoring systems for which the expected value of a guess beyond the test-taker's knowledge is negative, and the inequalities (8) and (11) provide this condition. However, in the actual testing situation, the only variance observed is that among the test-takers, and this variance is not only due to random guessing, but includes also differences in measured ability in the test-taker population. This is variance that should be conserved in the test situation. We shall therefore narrow down our choice for $p_{i}$ further.

\subsection{Relation between item and latent score}

Assume a test-taker knows a fraction $f$ of the material (distractors and keys; $f$ is directly proportional to the extent of the test-taker's factual knowledge in the domain tested). The expectation value of the points that she will get for an item, relative to a blank answer for which she is awarded $p_{c}$ points, is then $\sum_{i}^{c-1}\left(p_{i}-p_{c}\right) P_{i}(f, c)$ but in order for the points awarded to reflect the expected knowledge, this sum should equal $f$ (or, at any rate, $f$ times a constant). We thus have an equation,

$$
\sum_{i=1}^{c-1}\left(p_{i}-p_{c}\right) P_{i}(f, c)=f
$$

Since $P_{i}(f, c)$ is polynomial of degree $c$, one obtains from this equation a linear system of $c$ equations and $c-1$ unknowns by comparing coefficients in $f$. Since the system is overdetermined, we conclude that a perfectly linear correlation between the expected test score and our model for the latent score is unattainable for a multiple-choice test. This is the conclusion we drew earlier in a preceding section, but repeated here on more formal grounds.

To make headway, we pursue an alternative (albeit approximate) approach in which we consider that the test-taker knows a fraction $g / c$ of the answer options for an item, where $g=1, \ldots, c-1$, and then compute the expected score relative to the blank answer under the following assumption: if the key is known (which it is with probability $g / c$ ), 
the test-taker answers correctly for $p_{1}$ points; otherwise, she answers partially correctly for $p_{c-g}$ points. In this case, requiring that the expected score with respect to the blank answer equals the knowledge possessed, one obtains a linear system of $c-1$ equations of the form,

$$
\frac{c-g}{c}\left(p_{c-g}-p_{c}\right)+\frac{g}{c}\left(p_{1}-p_{c}\right)=\frac{g}{c}
$$

If one then sets $p_{c}=1 / c$, the solution becomes the scoring system of Zapechelnyuk (2015) [also independently reinvented by Otoyo and Bush (2018) on heuristic grounds], where in our notation $\epsilon_{i}=0$ for $i>1$ and $\epsilon_{1}=1$. The fact that $\epsilon_{i}=0$ for $i>1$ implies a weak violation of inequality (8), meaning both that the risk-neutral test-taker may guess (increased random variance) and that the latent score of the risk-averse test-taker may be underestimated.

Seeing as keeping the relation of the score with the fraction of knowledge possessed as linear as possible is desirable, one might inquire about other choices of $p_{c}$. One can easily verify that values of $p_{c}<1 / c$ will violate the condition that $\epsilon_{i}>0$ even further, but values of $p_{c}>1 / c$ lead to compliance $\left(\epsilon_{i}>0\right)$ and know only the limit set by inequality (11). Moreover, the point difference with respect to the blank answer remains the same, but the relative point gaps between consecutive types of answers change. Thus, in reality, the precise value of $p_{c}>1 / c$ will be dictated by the psychology of the test-takers (e. g., degree of risk aversion or risk seeking).

\section{Comparison of scoring systems for test items}

Having established our formalization of the rational test-taker and her latent score, we shall investigate different scoring systems for the test items for purposes of illustration and of comparison. These different systems correspond to how many points are awarded for different types of answers to one and the same item, the type of answer in turn being dictated by the rational test-taker that we consider in our formalization. For brevity, we will not consider every example which can be found in the literature, even if the mathematical approach is general enough. In the following, we let $m$ denote the number of distractors known to the test-taker and $c$ the total number of answer options on the multiple-choice test item.

In the first scoring system considered, corresponding to the typical "number correct" scheme, the test-taker is awarded $p_{1}$ points if either the key or all of the distractors are known. If not, $p_{1}$ points are awarded with a probability of $1 /(c-m)$. This situation corresponds to the rational test-taker guessing whenever in doubt, and answering correctly whenever certain.

In the second one, corresponding to a modified Zapechelnyuk scoring rule (with $\left.p_{c}>1 / c\right)$, points are awarded according to the following procedure: If either the key or all of the distractors are known, the test-taker is awarded $p_{1}$ points. If the key is not known, the test-taker is awarded $p_{c-m}$ points. The set $\left\{p_{i}\right\}$ is defined by solving the system of equations implicit in eq. (13) with $p_{c}>1 / c$. The precise value of $p_{c}$ has no effect on the behavior of our formalized, rational test-takers as long as it is greater than $1 / c$.

In the third one, proposed by Frandsen and Schwartzbach (2006), $p_{1} \ln (c)$ points are given if either the key is known or all distractors are and $p_{1} \ln (c /(c-m))$ points otherwise. In the original formulation, there is a variable point penalty for incorrect 
answers designed to nullify the expected score of guessing. We do not need to consider it explicitly here because the test-takers we model are not risk-seeking.

In the fourth one, corresponding to the popular "subset selection" scoring first proposed by Dressel and Schmid (1953), $p_{1}$ points are awarded if the test-taker knows the key or all of the distractors, and otherwise $p_{1}(1-(c-m-1) /(c-1))$ points are awarded. It must be pointed out that this scoring rule, as formulated in the original reference, strictly violates eq. (8) on several points. It is therefore implicitly assumed that a penalty for incorrect answers is also included in the scoring function so as to negate the expected value of all guesses.

In the last one, $p_{1}$ points are awarded for the correct answer, and a "very large" number is subtracted as a penalty for providing the wrong answer, meaning in our case that the test-taker will answer if she knows the answer with complete certainty and leave it blank otherwise for no points. For our purposes, we do not need to specify exactly how large this penalty is, but it is chosen at least as large as to make the expected score of a random guess on only two options negative.

For brevity, we shall distinguish whenever necessary between these scoring systems using the acronyms "NC" (number correct), "MZ" (Modified Zapechelnyuk), "FS" (Frandsen and Schwartzbach), "SS" (subset selection) and "NG" ("no guessing"), respectively.

A test is composed of several independent items, which we consider to be drawn randomly from a set of keys and distractors. We denote the score on an item by $S$, and the total score is then simply the sum of the score on each item (and the total variance is the sum of the individual variances). In what follows, we simply deal with a test composed of a single item for simplicity but without any loss of generality. Results reported for a fixed value of $f$ can be interpreted either as an average over all items on an infinite test for a single test-taker of said ability, or as the average over an infinite number of test-takers of fixed ability on a fixed item.

\subsection{Test-retest variance}

Among the rule systems considered above, only the NC scoring rule will generate test-retest variance for the rational test-taker. For the test-taker of aptitude $f$, the magnitude of this variance for a single item is given by,

$$
V_{\mathrm{trt}}(f, c)=p_{1}^{2} \sum_{i=2}^{c} \frac{(i-1) P_{i}(f, c)}{i^{2}}
$$

where $p_{1}$ is the point value of a correct answer. For a test item with $c=4$, this is a monotonously decreasing function of $f$, starting off at $f=0$ with its highest value of $0.1875 p_{1}^{2}$. The most pronounced test-retest variance is for $c=2$, for which the explicit function is

$$
V_{\mathrm{trt}}(f, 2)=p_{1}^{2} \frac{1-2 f+f^{2}}{4}
$$

This provides a baseline scale of the increased accuracy afforded by the suppression of guessing when compared to the second moments reported below. 


\subsection{Moments of the score function}

For completeness, we here present the formulae for the first and second moments of the score that follow when the rational test-taker maximizes her expected score under the different scoring rules defined above.

For the first model of score attribution, corresponding to "number correct" counting without any penalty for wrong answers, the first and second moments of $S$ are

$$
\left\langle S_{\mathrm{NC}}(f, c)\right\rangle=p_{1} \sum_{i=1}^{c} \frac{P_{i}(f, c)}{i}
$$

and

$$
\left\langle S_{\mathrm{NC}}(f, c)^{2}\right\rangle=p_{1}^{2} \sum_{i=1}^{c} \frac{P_{i}(f, c)}{i}
$$

For the second scoring system (Otoyo \& Bush, 2018; Zapechelnyuk, 2015, slightly modified to negate guessing), we have

$$
\left\langle S_{\mathrm{MZ}}(f, c)\right\rangle=\sum_{i=1}^{c} p_{i} P_{i}(f, c)
$$

and

$$
\left\langle S_{\mathrm{MZ}}(f, c)^{2}\right\rangle=\sum_{i=1}^{c} p_{i}^{2} P_{i}(f, c)
$$

For the third scoring rule considered (Frandsen \& Schwartzbach, 2006), we have

$$
\left\langle S_{\mathrm{FS}}(f, c)\right\rangle=p_{1} \sum_{i=1}^{c-1} \ln \left(\frac{c}{i}\right) P_{i}(f, c)
$$

and

$$
\left\langle S_{\mathrm{FS}}(f, c)^{2}\right\rangle=p_{1}^{2} \sum_{i=1}^{c-1} \ln \left(\frac{c}{i}\right)^{2} P_{i}(f, c)
$$

For the fourth system, "subset selection" (Dressel \& Schmid, 1953, suitably modified to eliminate guessing), we have

$$
\left\langle S_{\mathrm{SS}}(f, c)\right\rangle=p_{1} \sum_{i=1}^{c-1}\left(1-\frac{i-1}{c-1}\right) P_{i}
$$

as well as

$$
\left\langle S_{\mathrm{SS}}(f, c)\right\rangle=p_{1}^{2} \sum_{i=1}^{c-1}\left(1-\frac{i-1}{c-1}\right)^{2} P_{i}
$$




\begin{tabular}{l|r} 
& Pearson's $r$ \\
\hline NC & 0.9898 \\
NG & 0.9969 \\
SS & 0.9683 \\
FS & 0.9816 \\
MZ & 0.9898
\end{tabular}

Table 1. Calculated Pearson product-moment correlation coefficients between observed and latent scores rounded to four decimal places for the different scoring functions with $c=4$.

Finally, under the "no guessing" scoring, we have

$$
\left\langle S_{\mathrm{NG}}(f, c)\right\rangle=p_{1} P_{1}(f, c)
$$

and

$$
\left\langle S_{\mathrm{NG}}(f, c)^{2}\right\rangle=p_{1}^{2} P_{1}(f, c)
$$

With the exception of the NC scoring rule, these moments are computed on the assumption of no guessing on the test-taker's part. For MZ this entails a slight, and for SS a correspondingly large, implicit deviation from the scoring rule formulations in the original references to ensure that guessing is not a rational strategy.

\subsection{Linear correlation with latent score}

It is usually desirable to have a scoring system that gives as linear as possible a relation with the latent score in order to enhance and facilitate comparisons between test subjects. It also means that the score (with respect to the underlying ability) is given on an interval, as opposed to ordinal, scale. Even though the scoring systems that award partial credits cannot obtain this perfectly linear relation, they can still vary between themselves in the degree to which this objective is attained.

Whereas the rank correlation between $\langle S\rangle$ and $f$ is unity for all of the considered scoring rules (ensuring they are valid for rank sorting and constitute at least a true ordinal scale), the linear Pearson correlation coefficient differs slightly between them. Keeping $c=4$ as our test case, the calculated results are reported in Table 1 . In all cases, the greatest deviations from linearity are observed for $f$ close to unity (not shown), which is expected because higher-order polynomial terms in eq. (12) become important only at large $f$, being naturally suppressed for small $f$.

\subsection{Validity}

We consider the statistical agreement between the obtained score and the latent score $f$ as an indication of the validity of test under the different scoring rules.

The variance in $S$ on an item among test-takers of fixed ability $f$ is given by

$$
V(f, c)=\left\langle S(f, c)^{2}\right\rangle-\langle S(f, c)\rangle^{2}
$$

where $\langle\cdot\rangle$ denotes an arithmetic average. To obtain the population-averaged variance, 
we integrate over the latent-score distribution,

$$
\Sigma^{2}(c)=\int_{0}^{1} V(f, c) \phi(f) \mathrm{d} f
$$

where $\phi(f)$ is the probability density function for $f$ in the test-taker population. In the language of classical test theory, this variance is error variance (and not "true score" variance; vide infra) since it is the variance for scores for fixed ability. In addition, we define the expected error as

$$
\langle E(f, c)\rangle=\langle S(f, c)-S(0, c)\rangle-f\langle S(1, c)-S(0, c)\rangle
$$

and compute its variance

$$
\sigma_{\mathrm{E}}^{2}(c)=\int_{0}^{1}\langle E(f, c)\rangle^{2} \phi(f) \mathrm{d} f-\left[\int_{0}^{1}\langle E(f, c)\rangle \phi(f) \mathrm{d} f\right]^{2}
$$

across $f$ for the different scoring systems as per above. The variance $\sigma_{\mathrm{E}}^{2}$ represents the "Platonic true score" error variance, in that it is the variance of the deviation of the expected score from the value linearly predicted by the underlying ability. We now define a validity coefficient as that proportion of the total error that is not "Platonic true score" error variance, i.e.,

$$
\rho(c)=1-\frac{\sigma_{\mathrm{E}}^{2}(c)}{\Sigma^{2}(c)+\sigma_{\mathrm{E}}^{2}(c)}
$$

This coefficient is bounded between zero and unity and obtains its maximum when the prediction by the expectation never deviates from linearity. On the contrary, it obtains its minimum if there is no statistical uncertainty around erroneous predictions. In other words, we interpret a scoring rule to be "valid" if on average it predicts the $f$-score linearly, no matter how large the dispersion around this prediction (which we take to be captured by the reliability and the measurement precision).

Both variances are functions of the $f$-distribution chosen and to give arbitrary but clear indications of the effects of the different scoring systems, we consider a test with $c=4$ and two different choices for $\phi(f)$ : one "broad" distribution (Distribution I), which we take to be the uniform distribution for $f \in[0,1]$, and one "narrow" distribution (Distribution II), which we take to be the normal distribution with mean $\langle f\rangle=0.6$ and standard deviation $\sigma_{f}=0.1$. Refer to Table 2 for the results. In general, the computed validity is smaller for Distribution I than for Distribution II and this decline in the accuracy is mainly a consequence of sampling the ability distribution for $f$ close to unity.

\subsection{Reliability}

The total variance for the observed score is the sum of the variance in eq. (27), representing the contribution to the variance from test-takers of fixed ability, and the two 


\begin{tabular}{l|rr} 
& Distribution I & Distribution II \\
\hline NC & 0.9916 & 0.9995 \\
NG & 0.9900 & 0.9983 \\
SS & 0.9333 & 0.9917 \\
FS & 0.9624 & 0.9978 \\
MZ & 0.9784 & 0.9991
\end{tabular}

Table 2. Computed validity coefficients for $c=4$ rounded to four decimal places for two different distributions of the ability: one broad and one narrow. See text for details.

\begin{tabular}{l|rr} 
& Distribution I & Distribution II \\
\hline NC & 0.2593 & 0.0390 \\
NG & 0.4100 & 0.0639 \\
SS & 0.5338 & 0.0844 \\
FS & 0.5089 & 0.0807 \\
MZ & 0.4771 & 0.0756
\end{tabular}

Table 3. Computed reliability coefficients for a test item with $c=4$ rounded to four decimal places for two different distributions of the ability: one broad and one narrow. See text for details.

terms,

$$
\int_{0}^{1}\langle S(f, c)\rangle^{2} \phi(f) \mathrm{d} f-\left[\int_{0}^{1}\langle S(f, c)\rangle \phi(f) \mathrm{d} f\right]^{2}
$$

representing the "true score" variance, the "true score", $\langle S(f, c)\rangle$, being simply the expectation value of the observed score (Klein \& Cleary, 1969). Hence, the reliability coefficient, given as the ratio of true score variance to total variance is,

$$
r=\frac{\int_{0}^{1}\langle S(f, c)\rangle^{2} \phi(f) \mathrm{d} f-\left[\int_{0}^{1}\langle S(f, c)\rangle \phi(f) \mathrm{d} f\right]^{2}}{\int_{0}^{1} V(f, c) \phi(f) \mathrm{d} f+\int_{0}^{1}\langle S(f, c)\rangle^{2} \phi(f) \mathrm{d} f-\left[\int_{0}^{1}\langle S(f, c)\rangle \phi(f) \mathrm{d} f\right]^{2}}
$$

Note that this reliability coefficient is that which is computed as an average over parallel forms of the test with non-identical items; it is not a test-retest coefficient. There is thus a random element whether or not the test-taker knows the keys and distractors on the parallel form, but even if non-identical, the items are still equivalent from the perspective of the model.

Under the same conditions as the computed validity coefficients, the calculated results are reported in Table 2. All of the partial credits scoring functions exhibit increased reliability with respect to both NC and NG scoring. An increased reliability for NG vis-à-vis NC scoring is also apparent, but it does not quite reach up the level of the partial credits models. The modified SS scoring rule (with penalties added for guessing) exhibits the highest predicted reliability. It is to be stressed that without this important modification, its reliability would be lower, approaching that of NC.

The decrease in computed reliability when moving from the broad to the narrow ability distribution is a consequence of the diminished true score variance, leaving more of the variance to chance effects. For a perfectly homogeneous distribution where all the test-takers share the same ability, there is no true score variance at all and the reliability coefficient is zero. The highest reliabilities will be obtained for distributions that are weighted toward the upper level of $f$-ability, because then the chance effects 


\begin{tabular}{l|rr} 
& Distribution I & Distribution II \\
\hline NC & 0 & 0 \\
NG & 0 & 0 \\
SS & 0.35 & 0.38 \\
FS & 0.49 & 0.20 \\
MZ & 1.47 & 0.02
\end{tabular}

Table 4. Discrimination index, $d$, rounded to two decimals for $\Delta=\delta p / 10$ and $c=4$ for two different ability distributions: one broad and one narrow. See text for details.

are reduced.

\subsection{Discriminatory power}

Scoring items polytomously allows a finer discrimination among the observed scores compared to dichotomous scoring. In this section, we seek to quantify this added resolution. In the literature, the "discrimination" of a test item is usually taken to be its Pearson correlation with the total test score. This is not sufficient for our analysis. We want to quantify the probability that a single test-taker on a single item receives a fair score reflecting her latent ability as precisely as possible.

Let $\pi(s ; f, c)$ be the probability that a test-taker of ability $f$ achieves at least a score $s$ on an item with $c$ options. Clearly, we have

$$
\pi_{\mathrm{NG}}(s ; f, c)=P_{1}(f, c)
$$

and

$$
\pi_{\mathrm{NC}}(s ; f, c)=P(f, c)
$$

independently of $s$ for $s>0$ for any scoring system that does not award partial marks (the probability is unity for $s=0$ ). With the MZ system and no guessing, we have

$$
\pi_{\mathrm{MZ}}(s ; f, c)=\left\{\begin{array}{lr}
\sum_{i=1}^{c} P_{i}(f, c), & s \in\left[p_{c}, p_{c-1}\right) \\
\sum_{i=1}^{c-1} P_{i}(f, c), & s \in\left[p_{c-1}, p_{c-2}\right) \\
\vdots & \vdots \\
P_{1}(f, c), & s \geq p_{1}
\end{array}\right.
$$

and the cases for FS and for SS follow analogously.

Denote the point value of a blank answer by $p_{\text {blank }}$. Then we use the integral

$$
d=\int_{0}^{1} \mathrm{~d} f \frac{\pi\left(f \delta p+p_{\text {blank }} ; f, c\right)-\pi\left(f \delta p+p_{\text {blank }}+\Delta ; f, c\right)}{\Delta} \phi(f)
$$

as a measure of the discriminatory power of the scoring rule. Here $\delta p$ is the point difference between a fully correct and blank answer (usually this is $p_{1}-p_{\text {blank }}$ in the terminology of this paper, but for the FS rule, it is $p_{1} \ln c$ ) and $\Delta$ is a sensitivity parameter which we arbitrarily set $\Delta=\delta p / 10$. Higher values of $d$ indicate that scores are allotted more finely attuned with ability and with certainty. Results are given in Table 4. 


\begin{tabular}{l|rr} 
& Distribution I & Distribution II \\
\hline NC & 0.564 & 0.472 \\
NG & 0.414 & 0.488 \\
SS & 0.419 & 0.323 \\
FS & 0.476 & 0.398 \\
MZ & 0.544 & 0.480
\end{tabular}

Table 5. Population-averaged relative uncertainties of the observed scores on a test item with $c=4$ for two different ability distributions. See text for details.

As seen, the results are very sensitive to the underlying distribution, with a complete reversal of the ranking of the partial credits model between Distributions I and II. The small value for MZ for the narrow distribution centered on $f=0.6$ reflects its poor discriminatory power around $f \approx 0.6$. In fact, this scoring function is most discriminatory for $f<0.5$ and becomes essentially dichotomous above that.

\subsection{Relative precision}

The precision of the score reflects the information gained, in the sense that one is certain that the obtained score is correct (Mellenbergh, 1996), and this statistic is distinct from the reliability which measures the extent to which the obtained scores for the same test-taker in independent measurements are correlated. We compute it by normalizing the standard deviation at fixed ability by the expectation of the score, thus

$$
\text { relative uncertainty }=\frac{V(f, c)}{\langle S(f, c)\rangle-p_{\text {blank }}(c)}
$$

where $V(f, c)$ is given in eq. (26) and $p_{\text {blank }}(c)$ represents the score of a blank answer. This procedure yields a number that is more aptly termed "relative uncertainty" than "relative precision", since larger values correspond to less precision. Averaged results over Distributions I and II are given in Table 5.

Contrary to the case of the reliability, the precision is increased for the narrower distribution with respect to the broad uniform distribution. This is mostly a consequence of the reduced chance variation for test-takers of higher ability. Indeed, for the test-taker of ability $f=1$, the computed precision is perfect. As inferred from the results, in the lower-range of ability, the NG scoring rule yields the most precise relative measurements, but this advantage is lost with more knowledgeable test-takers. Across both distributions, the modified SS rule is arguably the most precise. Note also that the large relative uncertainties (of the order of 30-50\%) reported here are for a single test item. Unlike the reliability above, as the number of items is increased, the relative uncertainty decays asymptotically as an inverse square-root toward zero within the model.

\section{Conclusion}

Having considered a model where the test-taker is presumed to possess a definite set of "facts" that are reflected in the distractors and keys of the multiple-choice test, we have compared different scoring rules for the kind of multiple-choice test items for 
which partial knowledge is expected to contribute to the test-taking strategy. We find that there is no single clearly superior scoring rule among the ones tested, each having its own merits: the NG scoring rule produces scores with the highest linear correlation with the underlying construct, the modified SS rule yields the highest reliability and precision (its unmodified form does not suppress guessing), the FS rule yields the most consistent item-level discrimination across the ability range, and so on. The modified Zapechelnyuk rule (Zapechelnyuk, 2015) is highly linear and discriminatory for the lower end of the ability distribution. It also has the highest validity among the partial credits scoring rules. The virtues of this scoring system, as regards the reliability, have been noted by Otoyo and Bush (2018) in an empirical study comparing it to traditional NC scoring. Here, we replicate this finding theoretically, but also report that other partial credits scoring rules should yield even higher reliabilities, a finding which should be confirmed empirically.

According to our underlying model, which seems intuitively valid as far as the testing of factual knowledge is concerned (for instance, tests of vocabulary, geography, qualitative science concepts, etc.) the score on a multiple-choice test, at least as far as it is composed as a sum over scores on individual items, cannot map perfectly linearly to the underlying latent score, except for the smallest scores. This effect is different from the traditional "ceiling effect", as it is an artifact of the binomial probability distributions that govern the probabilities in the parallel multiple-choice test item and would not appear in the same guise on a constructed-response test.

We should also point out that the formal model that we have presented, and which is the basis for our analysis, relies on an axiomatic representation of factual knowledge and the probability of solving a multiple-choice test item. The approach is hence fundamentally different from polytomous Rasch (Andrich, 1978; Masters, 1982) and item-response models (Wu, De Laet, \& Janssen, 2019), which rely on statistical fitting, but similar to knowledge space theory (Doignon \& Falmagne, 1985, 2012) in its epistemological assumptions, of which it can be considered a limiting special case. Moreover, we stress that the partial credits scoring systems investigated are intended to suppress guessing a priori; they hence differ fundamentally from any approach (Birnbaum, 2008; Lee \& Bolt, 2018; Martín, Del Pino, \& De Boeck, 2006; Ramsay, Wiberg, \& Li, 2020) which attempts to account for guessing a posteriori. These methods are inherently unreliable whenever sample sizes are small and are hence not suitable for general classroom assessments. but limited to large-scale assessments.

We should also briefly mention the work of Espinosa and Gardeazabal (2010) who analyze the optimal penalty for incorrect guessing using computer simulations of a model based on item-response and decision theory. This allows them to model a testtaker who is not completely rational or risk neutral with respect to maximizing her expected score. The main finding is that the penalty for incorrect guessing should exceed the typical one which is usually taken to precisely negate the effect of blind guessing. As far as the MZ scoring rule is concerned, this means that $p_{c}$ should be chosen sufficiently large. As signaled in the Introduction, similar simulations within the framework of classical test theory are reported by Chica and Tárrago (2021) for the effect on the reliability of the assessment with partial credits. We have eschewed risk-taking as part of our analysis because it leads to added arbitrariness in the form of extra parameters and guessing will obviously always lead to a decrease of the test reliability.

In regards to this last point, the results presented here are not arbitrary in that they are exact results for test-takers with perfect compliance with their own self-interest and awareness of the limits of their knowledge. While there might be discrepancies 
in real-world settings, we can consider these results as a mathematical limiting case. Real-world testing situations will presumably approach this limit if the test-takers are clearly instructed that "guessing" in the sense of identifying more than one possible answer leads to an expected increase of their score, relative to guessing on a single answer. It is not altogether unlikely that this removes the "ethical dilemma" that apparently keeps some test-takers from guessing even when explicitly encouraged to do so (Traub \& Hambleton, 1972). Moreover, students have been found highly compliant with instructions not to guess even in the absence of any penalty scoring (Delgado, 2007). If both the score-optimal strategy and the instructions align, it is difficult to imagine anything but stronger compliance, especially if students are encouraged to be honest about their partial knowledge.

\section{References}

Akeroyd, M. (1982). Progress in Multiple Choice Scoring Methods, 1977/81. Journal of Further and Higher Education, 6(3), 86-90.

Andrich, D. (1978). A rating formulation for ordered response categories. Psychometrika, 43(4), 561-573.

Arnold, J., \& Arnold, P. (1970). On scoring multiple choice exams allowing for partial knowledge. The Journal of Experimental Education, 39(1), 8-13.

Ben-Simon, A., Budescu, D. V., \& Nevo, B. (1997). A comparative study of measures of partial knowledge in multiple-choice tests. Applied Psychological Measurement, 21(1), 65-88.

Betts, L. R., Elder, T. J., Hartley, J., \& Trueman, M. (2009). Does correction for guessing reduce students' performance on multiple-choice examinations? Yes? No? Sometimes? Assessment $\& 3$ Evaluation in Higher Education, 34(1), 1-15.

Birnbaum, A. (2008). Some latent trait models and their use in inferring an examinee's ability. In F. M. Lord \& M. R. Novick (Eds.), Statistical theories of mental test scores (chap. 17). Charlotteville, North Carolina: Information Age Publishing.

Bliss, L. B. (1980). A test of Lord's assumption regarding examinee guessing behavior on multiple-choice tests using elementary school students. Journal of Educational Measurement, 147-153.

Bradbard, D. A., Parker, D. F., \& Stone, G. L. (2004). An alternate multiple-choice scoring procedure in a macroeconomics course. Decision Sciences Journal of Innovative Education, 2(1), 11-26.

Budescu, D., \& Bar-Hillel, M. (1993). To guess or not to guess: A decision-theoretic view of formula scoring. Journal of Educational Measurement, 30(4), 277-291.

Burton, R. F., \& Miller, D. J. (1999). Statistical modelling of multiple-choice and true/false tests: ways of considering, and of reducing, the uncertainties attributable to guessing. $A s$ sessment $\mathcal{E}$ Evaluation in Higher Education, 24(4), 399-411.

Bush, M. (2001). A multiple choice test that rewards partial knowledge. Journal of Further and Higher education, 25(2), 157-163.

Chica, J. C., \& Tárrago, M. J. G. (2021). Estudio de la fiabilidad de test multirrespuesta con el método de Monte Carlo. Revista de Educación, 392, 63-95.

Coombs, C. H., Milholland, J. E., \& Womer, F. B. (1956). The assessment of partial knowledge. Educational and Psychological Measurement, 16(1), 13-37.

Delgado, A. R. (2007). Using the Rasch model to quantify the causal effect of test instructions. Behavior Research Methods, 39(3), 570-573.

Diamond, J., \& Evans, W. (1973). The correction for guessing. Review of Educational Research, $43(2), 181-191$.

Dirkzwager, A. (2003). Multiple evaluation: A new testing paradigm that exorcizes guessing. International Journal of Testing, 3(4), 333-352. 
Doignon, J.-P., \& Falmagne, J.-C. (1985). Spaces for the assessment of knowledge. International journal of man-machine studies, 23(2), 175-196.

Doignon, J.-P., \& Falmagne, J.-C. (2012). Knowledge spaces. Springer Science \& Business Media.

Dressel, P. L., \& Schmid, J. (1953). Some modifications of the multiple-choice item. Educational and Psychological Measurement, 13(4), 574-595.

Espinosa, M. P., \& Gardeazabal, J. (2010). Optimal correction for guessing in multiple-choice tests. Journal of Mathematical psychology, 54 (5), 415-425.

Finetti, B. d. (1965). Methods for discriminating levels of partial knowledge concerning a test item. British Journal of Mathematical and Statistical Psychology, 18(1), 87-123.

Frandsen, G. S., \& Schwartzbach, M. I. (2006). A singular choice for multiple choice. $A C M$ SIGCSE Bulletin, 38(4), 34-38.

Frary, R. B. (1982). A simulation study of reliability and validity of multiple-choice test scores under six response-scoring modes. Journal of Educational Statistics, 7(4), 333-351.

Gibbons, J. D., Olkin, I., \& Sobel, M. (1979). A subset selection technique for scoring items on a multiple choice test. Psychometrika, 44(3), 259-270.

Horst, P. (1933). The difficulty of a multiple choice test item. Journal of educational psychology, $24(3), 229$.

Klein, D. F., \& Cleary, T. A. (1969). Platonic true scores: Further comment. Psychological bulletin, $71(4), 278$.

Lee, S., \& Bolt, D. M. (2018). An alternative to the 3pl: Using asymmetric item characteristic curves to address guessing effects. Journal of Educational Measurement, 55 (1), 90-111.

Lesage, E., Valcke, M., \& Sabbe, E. (2013). Scoring methods for multiple choice assessment in higher education-Is it still a matter of number right scoring or negative marking? Studies in Educational Evaluation, 39(3), 188-193.

Lord, F. M. (1975). Formula scoring and number-right scoring. Journal of Educational Measurement, $7-11$.

Martín, E. S., Del Pino, G., \& De Boeck, P. (2006). Irt models for ability-based guessing. Applied Psychological Measurement, 30(3), 183-203.

Masters, G. N. (1982). A rasch model for partial credit scoring. Psychometrika, 47(2), $149-174$.

Medawela, R. S. H. B., Ratnayake, D. R. D. L., Abeyasinghe, W. A. M. U. L., Jayasinghe, R. D., \& Marambe, K. N. (2018). Effectiveness of "fill in the blanks" over multiple choice questions in assessing final year dental undergraduates. Educación Médica, 19(2), 72-76.

Mellenbergh, G. J. (1996). Measurement precision in test score and item response models. Psychological Methods, $1(3), 293$.

Otoyo, L., \& Bush, M. (2018). Addressing the shortcomings of traditional multiple-choice tests: subset selection without mark deductions. Practical Assessment, Research, and Evaluation, $23(1), 18$.

Papenberg, M., Diedenhofen, B., \& Musch, J. (2021). An experimental validation of sequential multiple-choice tests. The Journal of Experimental Education, 89(2), 402-421.

Persson, R. A. X. (2021). Theoretical evaluation of partial credit scoring of the multipleresponse test item. PsyArxiv. Retrieved from https://psyarxiv.com/g5wmb/

Prieto, G., \& Delgado, A. R. (1999). The effect of instructions on multiple-choice test scores. European Journal of Psychological Assessment, 15(2), 143.

Ramsay, J., Wiberg, M., \& Li, J. (2020). Full information optimal scoring. Journal of educational and behavioral statistics, 45(3), 297-315.

Rasch, G. (1960). Probabilistic models for some intelligence and attainment tests. Copenhagen, Denmark: Danmarks Paedagogiska Institut.

Shuford, E. H., Albert, A., \& Massengill, H. E. (1966). Admissible probability measurement procedures. Psychometrika, 31(2), 125-145.

Slepkov, A. D., \& Godfrey, A. T. (2019). Partial credit in answer-until-correct multiple-choice tests deployed in a classroom setting. Applied Measurement in Education, 32(2), 138-150.

Traub, R. E., \& Hambleton, R. K. (1972). The Effect of Scoring Instructions and Degree 
of Speededness on the Validity and Reliability of Multiple-Choice Tests. Educational and Psychological Measurement, 32(3), 737-758.

Traub, R. E., Hambleton, R. K., \& Singh, B. (1969). Effects of promised reward and threatened penalty on performance of a multiple-choice vocabulary test. Educational and Psychological Measurement, 29(4), 847-861.

Vanderoost, J., Janssen, R., Eggermont, J., Callens, R., \& De Laet, T. (2018). Elimination testing with adapted scoring reduces guessing and anxiety in multiple-choice assessments, but does not increase grade average in comparison with negative marking. PloS one, 13(10), e0203931.

Wilcox, R. R. (1981). Solving measurement problems with an answer-until-correct scoring procedure. Applied Psychological Measurement, 5(3), 399-414.

Wu, Q., De Laet, T., \& Janssen, R. (2019). Modeling partial knowledge on multiple-choice items using elimination testing. Journal of Educational Measurement, 56 (2), 391-414.

Zapechelnyuk, A. (2015). An axiomatization of multiple-choice test scoring. Economics Letters, 132, 24-27.

Zimmerman, D. W., \& Williams, R. H. (1965). Effect of chance success due to guessing on error of measurement in multiple-choice tests. Psychological Reports, 16(3), 1193-1196.

Zimmerman, D. W., \& Williams, R. H. (2003). A new look at the influence of guessing on the reliability of multiple-choice tests. Applied Psychological Measurement, 27(5), 357-371.

\section{Appendix A. Derivation of Eqs (2) and (3)}

\section{A.1. Derivation of $P_{k}(f, c)$ for $k>1$}

The probability $P_{k}(f, c)$ with $k>1$ is directly proportional to the probability of the test-taker knowing all but $k$ alternatives. The probability that $k$ of the alternatives are unknown and $c-k$ are known is $(1-f)^{k} f^{c-k}$ multiplied by the number of groupings with $k$ elements among the answer options, viz. the binomial coefficient

$$
\left(\begin{array}{l}
c \\
k
\end{array}\right)
$$

However, the probability that among these $c-k$ known alternatives, none is actually the key is $\left(1-c^{-1}\right)\left(1-(c-1)^{-1}\right) \cdots\left(1-(k+1)^{-1}\right)$. When the probability is corrected for this, we obtain eq. (3).

\section{A.2. The case $k=1$}

Let us first determine the contributions to $P_{1}(f, c)$ which include the cases where the key is known. Assuming $i$ distractors are known, then these probabilities are given by $f^{i+1}(1-f)^{c-i-1}$ multiplied by the binomial coefficient

$$
\left(\begin{array}{c}
c-1 \\
i
\end{array}\right)
$$

In addition to these probabilities, there is the special case of all distractors being known but not the key. The associated probability is $f^{c-1}(1-f)$. The sum of all these contributions yields eq. (2). 
The attentive reader will have noticed that eq. (2) may be simplified to,

$$
P_{1}(f, c)=f+(1-f) f^{c-1}
$$

corresponding to the sum of the two probabilities that the test-taker either knows the key or, barring that, all of the distractors (which are the only two ways that she may provide the correct answer with certainty). 